\title{
Reconstruction of the Polarization Ellipse of the EM Field of Telecommunication and Broadcast Antennas by a Fast and Low-Cost Measurement Method
}

\author{
Wout Joseph, Leen Verloock, and Luc Martens, Member, IEEE
}

\begin{abstract}
A low-cost measurement method for the extraction of the relative phases of the field of base station and broadcast antennas is presented. Our purpose is to determine the polarization of the field at a measurement site using magnitude measurements only. By determining six amplitude components using a spectrum analyzer, the polarization ellipse of the field can be obtained. This low-cost method can be used for outdoor measurements, which is not possible with network analyzers. Using this method and thus knowing the polarization of the incident field we are able to determine more accurately the actual electromagnetic power absorbed in people at a measurement site.
\end{abstract}

Index Terms-Base station antenna, electromagnetic field, measurement, measurement probe, phase extraction, polarization, specific absorption rate (SAR), telecommunication and broadcast antenna.

\section{INTRODUCTION}

$\mathbf{T}$ $\mathrm{O}$ determine whether the exposure of broadcast and telecommunication antennas complies with the safety standards, the electromagnetic fields around the antenna must be determined and be compared to the reference levels [1]. The reference levels are formulated for maximum coupling between the incident field and the human body. This coupling is dependent on the polarization of the field: $E$-polarized (incident electric field is parallel to the major axis of a human body) incident plane waves result in the highest whole-body specific absorption rate (SAR) values [2]. Knowing the polarization of the field allows us to more accurately determine the actual SAR using a correct model of the human body. The polarization of the incident (electric and magnetic) field can be determined if one knows the relative phases of all the orthogonal components of the field.

The relative phases can be measured using a network analyzer (NWA) but this is expensive and can not be used for outdoor measurements of, e.g., base station antennas. Our objective is to develop a low-cost measurement method to determine the magnitude and polarization of the electromagnetic field of an antenna immediately at a measurement site. Field measurements around broadcast and telecommunication antennas are usually

Manuscript received October 4, 2004; revised January 31, 2006. This work was supported by the Belgian Federal Science Policy Office under the Standardization Programmes and the Interdisciplinary Institute for BroadBand Technology (IBBT).

The authors are with the Department of Information Technology, Ghent University, B-9050 Gent, Belgium (e-mail: wout.joseph@intec.UGent.be; leen.verloock@intec.UGent.be; luc.martens@intec.UGent.be).

Digital Object Identifier 10.1109/TEMC.2006.873851 performed using a spectrum analyzer (SA). In [3]-[7], three orthogonal magnitudes are measured and from these three magnitudes $\left(E_{i}, i=1,2,3\right)$ the total field $\left(E_{\mathrm{tot}}=\sqrt{E_{1}^{2}+E_{2}^{2}+E_{3}^{2}}\right)$ is obtained. But using this method, the polarization cannot be obtained. When a SA is used, only power measurements are possible. Therefore, we use the algebraic method of [8] and [9] for the reconstruction of the relative phases, which offers substantial reduction in computational time over methods that use nonlinear optimization [10]. These nonlinear optimization methods are too slow for real-time application because they are based on iterative schemes. By determining six magnitudes, the relative phases can be obtained [8], [9]. Up to now this method was only theoretically described and was not yet applied to electromagnetic field measurements.

Other interesting approaches for the "phase-less" calculations of the SAR are described in [11] and [12]. We investigate in this paper an electromagnetic-field measurement method using only six magnitude components and determine the SAR with finitedifference time domain (FDTD) simulations (see further), while in papers [11] and [12] a fast calculation method for the SAR is described. The data obtained with our measurement method could also be used for fast and accurate calculation of the SAR with the method of [11] and [12]. This paper and [11] and [12] are complementary to each other.

First, the method to obtain the relative phases will be described in Section II. The practical implementation of the polarization extraction method is discussed in Section III. We validate the method in Sections IV and V. The validation with free-space electromagnetic simulations and NWA measurements using our de-embedding technique developed in [13] is discussed in Section IV. To experimentally validate our method using a SA, we compare the results with NWA measurements in Section V. Next, an outdoor application of the method to the characterization of fields around antennas is described in Section VI. The reconstruction of the polarization ellipse is applied to the far field of an antenna in a rural environment. Finally, the conclusions are presented in Section VII.

\section{TheORY OF THE POlarizATION EXTRACTION MethoD}

We use an algebraic method [8], [9] for the reconstruction of the relative phases. The method is based on the determination of $\mathrm{n}$ magnitudes of components in a $n$-dimensional orthogonal coordinate system and at least $2 n-3$ additional amplitude measurements in different directions. Thus in total $3(n-1)$ magnitude measurements are necessary to determine the relative 


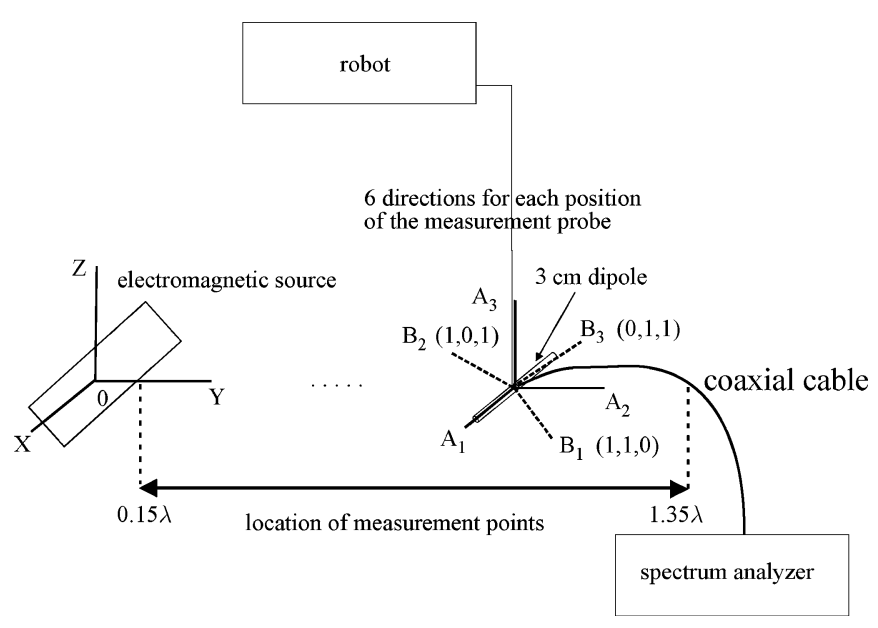

Fig. 1. Configuration for spectrum analyzer measurements.

phases. For $n=3$ ( 3 dimensions) this results in six magnitude measurements. Let $A_{1}, A_{2}$, and $A_{3}$ be the magnitudes of the sinusoidally time-varying components (represented by a complex vector $\boldsymbol{Z}$ ) of a real vector $\boldsymbol{X}=\boldsymbol{R e}\left\{\mathbf{Z e}^{\mathrm{j} \omega \mathrm{t}}\right\}$ in an orthogonal coordinate system. Let $B_{1}, B_{2}$, and $B_{3}$ be the magnitudes of $\boldsymbol{X}$ in three additional directions specified by the unit vectors $\boldsymbol{N}_{1}, \boldsymbol{N}_{2}$, and $\boldsymbol{N}_{3}$. [8] and [9] show that the additional magnitudes $B_{1}, B_{2}$, and $B_{3}$ obtained by rotating the measurement probe through other arbitrary angles cannot lead to a unique reconstruction of the relative phases. But when $B_{1}, B_{2}$, and $B_{3}$ are determined in the directions $(1,1,0),(1,0$, $1)$, and $(0,1,1)$ with respect to the Cartesian coordinate system, then a unique reconstruction is obtained. These directions are named the three standard directions.

Once the relative phases are known, the polarization ellipse can be determined. If we define the complex vector $\mathbf{Z}$ as $Z_{i}=$ $A_{i} e^{j \phi_{i}}(i=1,2,3)$, the semi-major axis $\boldsymbol{V}$ and semi-minor axis $\boldsymbol{R}$ of the ellipse will then have components $V_{i}$ and $R_{i}(i=$ $1,2,3)$ defined by

$$
V_{i}=\mathrm{A}_{i} \cos \left(\phi_{i}-\zeta\right)
$$

and

$$
R_{i}=\mathrm{A}_{i} \sin \left(\phi_{i}-\zeta\right)
$$

where

$$
\zeta=\frac{1}{2} \arg \left(\mathbf{Z}^{\mathrm{t}} \mathbf{Z}\right)
$$

\section{PRACTICAL IMPLEMENTATION OF THE POLARIZATION EXTRACTION METHOD}

\section{A. Setup for Lab Measurements With Robot}

We use a robot with an accuracy of $0.025 \mathrm{~mm}$ to position and rotate the measurement probe. We connect the SA with the measurement probe. A $15-\mathrm{cm}$ dipole will be used as Tx. The measurement configuration is shown in Fig. 1.

For the lab measurements with the robot, we have designed dipoles with a length of $3 \mathrm{~cm}$ with identical holders. We select these 3-cm dipoles because they deliver electric far- and nearfield measurements with a low disturbance (lower than 5\%) and have a sufficient sensitivity for practical measurements at 900 and $1800 \mathrm{MHz}$ (typical GSM frequencies) in both near and far field of the electromagnetic source (see [14]). The radius of the wire of the $3-\mathrm{cm}$ dipole is $1.8 \mathrm{~mm}$. We use the same balun and cables for all measurement probes. As the robot can only rotate a probe around a vertical axis, we had to come up with the following solution to measure the components of the field. For the dipole length of $3 \mathrm{~cm}$, we constructed two identical probes: one with an angle of $54.74^{\circ}$ and one with an angle of $35.26^{\circ}$ with the rotation axis, respectively. If the measurement probe is mounted at an angle of $54.74^{\circ}$, respectively $35.26^{\circ}$ with respect to the rotation axis, we obtain the magnitudes $A_{1}, A_{2}$, and $A_{3}$, respectively $B_{1}, B_{2}$, and $B_{3}$, by rotating the probe each time $120^{\circ}$. Although two probes have the same length, they will of course not be perfectly identical due to fabrication differences. As the two measurement probes are calibrated separately using a three-antenna method [15]-[17] these differences can be accounted for in the calibration. By performing the measurements with the two probes each immediately after the other at a measurement location and by using the robot to position and to rotate the measurement probe, we are able to minimize the inaccuracies when measuring the six required components to obtain the relative phases.

For validation of the method, this setup can be used either with a SA or a NWA.

\section{B. Setup for Outdoor Measurements}

In this section, the setup for practical measurements for compliance testing is described. We will extend the measurements of three orthogonal components to the determination of six magnitudes. Using this new setup and the method described in Section II, it is our objective to extract at real-time and at low cost the magnitudes and polarization of the fields.

The measurements are performed with an HP8561B spectrum analyzer. The use of a SA for the measurements makes it possible to identify the individual sources of exposure and to make accurate and sensitive measurements. Moreover, only a SA can be used for outdoor measurements. A NWA-which is even more expensive-for example cannot be used for outdoor compliance measurements of antennas because the source is mostly not accessible. Instead of a robot we use two holders with appropriate angles with the rotation axis to rotate the electric or magnetic field probes manually (see Fig. 2). The measurement system is also able to perform measurements as a function of the height above the ground.

We use a conical dipole antenna with a frequency range of $80 \mathrm{MHz}$ to $2.5 \mathrm{GHz}$ for the electrical field measurements. For the magnetic-field measurements, a split-shield loop antenna with diameter of $5 \mathrm{~cm}$ and thickness of $0.5 \mathrm{~mm}$ has been designed. The split-shield loop antenna is chosen to reject the contribution of the electric field to the magnetic-field measurement [18], [19].

\section{Spectrum Analyzer Measurements}

We can determine $A_{1}, A_{2}, A_{3}$ and $B_{1}, B_{2}, B_{3}$ by performing power measurements with the SA and using the antenna factor of the measurement probe. Fig. 1 shows the configuration for 


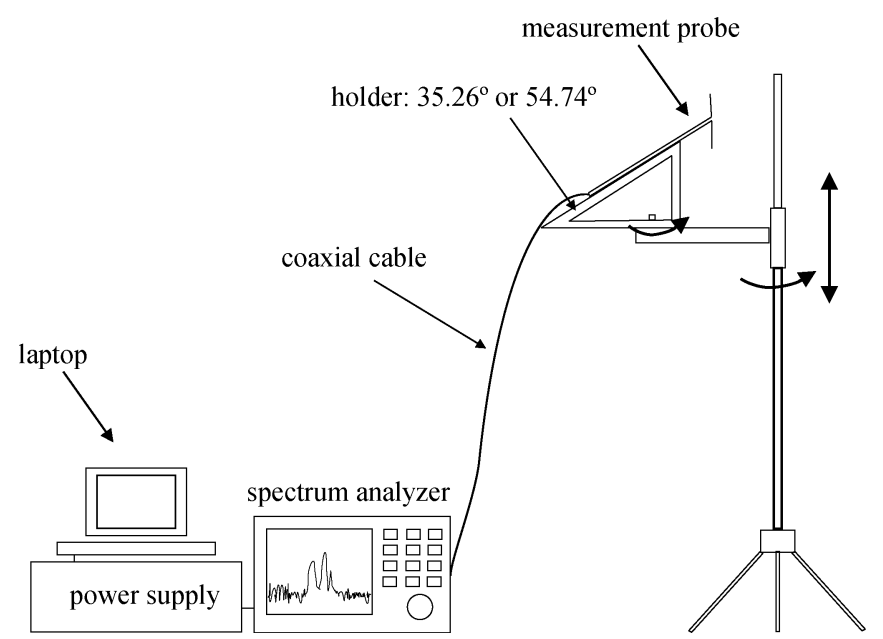

Fig. 2. Setup for outdoor measurement with a spectrum analyzer.

the SA measurements and the direction of the six components that have to be determined to obtain the relative phases.

When magnitude measurements are performed using the SA, the power at the investigated frequencies is displayed. These powers are converted into field values using the following formula:

$$
X_{i}^{\text {meas }}=\frac{1}{\sqrt{20}} \times 10^{\left(\frac{\mathrm{AF}_{\mathrm{X}}+\left(P^{\text {meas }}\right)_{\mathrm{i}}+L}{20}\right)}
$$

with

$X_{i}^{\text {meas }} \quad$ magnitude of component $i(i=1,2, \ldots, 6)$ of the electric $(\mathrm{X}=E)$ or magnetic field $(X=H)$;

$\left(P^{\text {meas }}\right)_{i}$ power measured with the $\mathrm{SA}$ in $\mathrm{dBm}$ corresponding to component $i(i=1,2, \ldots, 6)$;

$\mathrm{AF}_{X} \quad$ antenna factor [see (5)] of the $E$-field probe (e.g., dipole, $X=E$ ) or $H$-field probe (e.g., loop probe, $X=H$ );

$L \quad$ cable loss at the investigated frequency.

The antenna factor is defined as follows:

$$
\mathrm{AF}=20 \log \left(\frac{E^{i}}{V}\right)[d B(1 / m)]
$$

where $E^{i}$ is the electric field incident on the antenna to be calibrated, and $V$ is the voltage developed across the output of the antenna to be calibrated. Cable losses have to be taken into account when performing calibration and measurements. ANSI C63.5 recommends that only horizontal polarization should be used for antenna calibration [20].

1) Applicability to Continuous Wave, FM, and GSM Signals: The two holders (outdoor) or the two identical probes (in the lab) to determine the different magnitude components are positioned at the same location. Thus, the holders or probes have to be changed and the measurement probes have to be rotated, which results in performing measurements at a different time. The measurement time may thus influence the results.

For the lab validation, we use a continuous wave $(\mathrm{CW})$ signal that is constant in time and which means that the measurement time does not influence the results.
We will analyze in this paper an FM signal and a GSM signal (Section VI). The magnitude of the FM signal is almost constant in time thus minimizing this influence. For the GSM signal, we analyze the $\mathrm{BCCH}$ (broadcast control) channel that will also remain almost constant in time. Again, the influence of changing the holders will be limited. Moreover, for these compliance measurements, we use the maximum-hold setting of the SA to determine the maximum level of each signal in time. We assume thus that during the measurement time all signals in the measurement band reach a maximum. The longer the measurement time, the higher the probability the maximum value will be encountered.

The FM and GSM signals have a certain bandwidth and are not sinusoidal. GSM uses as modulation technique Gaussian Minimum Shift Keying (GMSK). With the appropriate SA settings, the power of the different magnitudes of the field components is then determined, e.g., we use a $300-\mathrm{kHz}$ resolution filter for the measurement of the GSM signal (the resolution filter of $300 \mathrm{kHz}$ is the smallest filter of the HP 8561B spectrum analyzer that can contain an entire $200-\mathrm{kHz}$ GSM frequency channel). The appropriate selection of the SA settings and the influence of the resolution filter are described in [21] and [22]. For the determination of the SAR using FDTD simulations (see Section VI), we further assume that all the power in the channel can be added to one single carrier frequency. The FDTD simulation is then performed at this carrier frequency and the SAR in a phantom can be obtained. We assume that the influence of the width of the narrowband channel will be limited for the determination of the whole-body SAR.

2) Applicability to UMTS Signals: For exposure assessment, a universal mobile telecommunications system (UMTS) signal will mainly differ from a GSM signal by the use of Wideband CDMA (code division multiple access) instead of TDMA/FDMA (time- and frequency-division multiple access) for GSM and the larger occupied signal bandwidth. The WCDMA based UMTS channel is approximately $5-\mathrm{MHz}$ wide. The UMTS frequency bands are $1920-1980 \mathrm{MHz}$ and 2110 $2170 \mathrm{MHz}$.

In UMTS, transmit power is adapted every $0.67 \mathrm{~ms}$ (rate of 1500 times per second) within a range of 0.5 to $3 \mathrm{~dB}$. The optimal measuring period should be chosen equally to two times the power control period of $0.67 \mathrm{~ms}$ [22]-[25]. The total sweep time over 601 frequency bins of the HP 8561B spectrum analyzer is then set to be $601 \times 2 \times 0.67 \mathrm{~ms}=0.8 \mathrm{~s}$ (using the maximum hold mode, the measurement consists of several such time sweeps). The resolution filter can be chosen equally to, e.g., $300 \mathrm{kHz}$. To measure the maximum level of each signal in time, the maximum-hold setting of the SA has to be used again. However, in maximum-hold mode where the positive-peak detector is used, the electromagnetic field strength will be overestimated due to the noise-like properties of the WCDMA signal (chip rate of $3.84 \mathrm{MChips} / \mathrm{s})$. Because the maximum occurred value of a noise-like signal is not a good measure for the signal strength of noise, a correction factor depending on the resolution filter must be used [21], [23], [25]. Since the worst-case root mean square (RMS) exposure-that is the maximum possible exposure on a certain location-has to be determined for compliance testing, 
this correction factor is the minimum ratio between the level measured by the positive-peak detector and the RMS level of the signal for all channel configurations [26].

Using these settings of the SA, the method could be applied to the UMTS signal. We further assume that during the measurement time, the UMTS signal in the measurement frequency band reaches a maximum and that the measured power of UMTS signal can be added to a single carrier for the determination of the SAR. We assume thus that the influence of width of the $5-\mathrm{MHz}$ channel is limited for the estimation of the actual whole-body SAR.

\section{Comparison Parameters for True and Measured Values}

First, the true field that should be measured is determined with the NWA (determined on the basis of three orthogonal components with magnitudes and phases of the $\mathrm{S}_{21}$-parameters) in the lab. We will note this as $X^{\text {true }}(X=E$ or $H)$, the true field. Next, six magnitude measurements are performed with the SA at each measurement position. Then the electric field amplitude of each component is derived using (4) and the relative phases are extracted using the methods of [8] and [9]. Finally, the extracted field using the SA is compared with the true field measured with the NWA. For this comparison, we define the maximum field $X_{\max }=\sqrt{V_{1}^{2}+V_{2}^{2}+V_{3}^{2}}(X=E$ or $H)$ as the magnitude of $\boldsymbol{V}$, the vector of the semi-major axis of the polarization ellipse [see (1)] and the minimum field $X_{\min }=$ $\sqrt{R_{1}^{2}+R_{2}^{2}+R_{3}^{2}}(X=E$ or $H)$ as the magnitude of $\boldsymbol{R}$, the vector of the semi-minor axis of the polarization ellipse [see (2)]. Further, $\boldsymbol{u}_{\boldsymbol{V}}$ and $\boldsymbol{u}_{\boldsymbol{R}}$ are noted as the unit vectors along $\boldsymbol{V}$ and $\boldsymbol{R}$, respectively. Using these parameters we can define deviations that quantify the accuracy of the extraction of the polarization ellipse (with the SA) with respect to the result obtained from NWA measurements. The relative deviation $\chi[\%]$ of the maximum/minimum field measured by the probe and SA with respect to the true maximum/minimum field (determined with NWA) is determined as follows:

$$
\chi_{y}=100 \frac{\left|X_{y}^{\text {true }}-X_{y}^{\text {meas }}\right|}{X_{y}^{\text {true }}}
$$

with $y=\max$ or min, meas $=$ measured with, e.g., SA.

Furthermore, we define $\Delta_{V}=\left|\mathbf{u}_{V}^{\text {true }}-\mathbf{u}_{\boldsymbol{V}}^{\text {meas }}\right|, \Delta_{\boldsymbol{R}}=$ $\left|\mathbf{u}_{\boldsymbol{R}}^{\text {true }}-\mathbf{u}_{\boldsymbol{R}}^{\text {meas }}\right|$, and the ellipticity $\kappa$ as

$$
\kappa=\frac{X_{\min }}{X_{\max }}
$$

with $X=E$ or $H$. The smaller $\kappa$ the more linearly the field is polarized. For $\kappa=1$, the field is circularly polarized.

\section{VALIDATION OF THE METHOD}

In this section we verify the applicability of the method of [8] and [9] and of the theory described in Section II, using NWA measurements and simulations with the electromagnetic software NEC-Win-Pro (based on the method of moments, MoM) in free space.

\section{A. Configuration}

We connect the SA with the measurement probe and inject a CW signal in the transmitting antenna (Tx) with a Rohde \& Schwarz signal generator (SMP 22). The Tx is a $15-\mathrm{cm}$ long dipole (about $\lambda / 2$ at $900 \mathrm{MHz}$ ) radiating at $900 \mathrm{MHz}$. The measurement probe is the $3-\mathrm{cm}$ long dipole. The centers of the Tx and measurement probe are positioned at the same height. The measurements are performed from $0.15 \lambda$ to $1.0 \lambda$ along the $y$-direction apart from the Tx at $\mathrm{x}=1.5 \mathrm{~cm}$ (see Fig. 1). We perform simulations at $x=1.5 \mathrm{~cm}$ because $E_{\min }$ will at this $x$-value not be zero in contrary to $x=0 \mathrm{~cm}$ where $E_{\text {tot }}=E_{\max }$, and thus $E_{\min }=0 \mathrm{~V} / \mathrm{m}$. The measurements and simulations are performed with a spatial grid of $1 \mathrm{~cm}$, smaller than $\lambda / 10=$ $3.3 \mathrm{~cm}$ at $900 \mathrm{MHz}$.

\section{B. Validation With Electromagnetic Simulations}

We perform simulations with the electromagnetic software NEC-Win-Pro in free space. The extended thin-wire kernel of the NEC-program is used because in some of the simulations, the ratio of the segment length to wire radius is small. Further, an applied $E$-field source model is used and the length of the source segment is made equal to the length of the other segments. The $E$-field source model corresponds to an applied voltage over the source segment.

We first perform NEC simulations of the true electric field, noted as $E_{\text {max }}^{\text {true }}$ and $E_{\text {min }}^{\text {true }}$. Then we execute NEC simulations of the measurement configuration. To this end, we first simulate the calibration of the measurement probe in the far field. Next, we perform for each "measurement" position six magnitude simulations rotating the measurement probe in NEC, and then, we determine $E_{\max }^{\operatorname{sim}}$ and $E_{\min }^{\operatorname{sim}}$ using the algorithm of [8]. Finally, we compare the results of the simulated measurement with the true simulated values (see Section IV-D).

\section{Validation With NWA}

Free-space simulations are compared with free-space measurements. We accomplish this by performing NWA measurements of six magnitude components, using a robot in an indoor open-labsite surrounded by absorbers and by applying our technique developed in [13]. Because of the nonanechoic property of the measurement site we have to take into account residual reflections. To this end, we perform a de-embedding step using the inverse fost Fourier transform (FFT) and a time-domain gating technique [27] to eliminate these residual reflections.

We use a Rohde \& Schwarz ZVR network analyzer. The measurement configuration for NWA measurements is shown in Fig. 3. We consider the combination of Tx and measurement probe as a two-port "circuit."

For each of the six magnitude components $\left(\left|\mathrm{S}_{21}\right|_{i}, \boldsymbol{i}=\right.$ $1,2, \ldots, 6)$ measured with the NWA, we eliminate the residual reflections. To determine the magnitudes of the field components with the NWA, the $\mathrm{S}_{21}$-parameters of two-port circuits are measured for each component. The field of a component at 


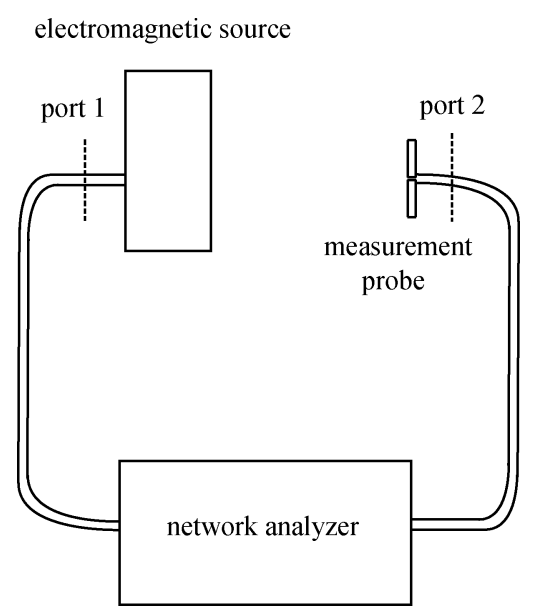

Fig. 3. Combining transmitting antenna and measurement probe as two-port circuit for network analyzer measurements.

a certain measurement point can be expressed as [13]

$$
\mathrm{X}_{i}^{\text {meas }}=\sqrt{50 \mathrm{P}_{i}} 10^{\frac{\mathrm{AF}_{\mathrm{X}}}{20}}\left|\mathrm{~S}_{21}\right|_{i}
$$

where

$\mathrm{X}_{i}^{\text {meas }}$ magnitude of component $i(i=1,2, \ldots, 6)$ of the electric $(X=E)$ or magnetic field $(X=H)$;

$\mathrm{P}_{\mathrm{i}} \quad$ available input power;

$\mathrm{AF}_{\mathrm{X}} \quad$ antenna factor of the $E$-field probe (e.g., dipole, $X=$ $E$ ) or H-field probe (e.g., loop probe, $X=H$ );

$\left|\mathrm{S}_{21}\right|_{i}$ magnitude of $\mathrm{S}_{21}$ corresponding to the component $i(i=1,2, \ldots, 6)$.

Using the algorithm of [8], $X_{\max }^{\text {meas }}$ and $X_{\text {min }}^{\text {meas }}$ are determined from $X_{i}^{\text {meas }}$.

\section{Comparison of True and Measured Polarization}

We investigate the configuration (see Section IV-A) with the Tx radiating at $900 \mathrm{MHz}$. Figs. 4 and 5 show $E_{\max }$ and $E_{\min }$ for the simulations and measurements as function of the distance from the source/ $\lambda$ at $900 \mathrm{MHz}$.

The extracted $E_{\max }$ agrees excellently with the simulated $E_{\max }^{\text {true }}$. The maximum and average relative deviation $\chi_{\max }$ (true $=$ NEC free-space simulation) for the simulation of the measurements are $0.4 \%$ and $0.2 \%$ and respectively $6.4 \%$ and $2.6 \%$ for the NWA measurement. The relative deviations $\chi_{\min }$ are larger for larger distances from the source due to the very small values of $E_{\min }$ in free space. Up to $\lambda / 2$, the maximum and average relative deviation for the simulation of the measurements are $9.3 \%$ and $4.5 \%$ and respectively $38.1 \%$ and $18.5 \%$ for the NWA measurement. For larger distances these deviations can go up to $2.5 \mathrm{~dB}$. These deviations are due to measurement errors of the NWA, calibration errors, positional and rotational errors, and the imperfect identical probes. Also residual reflections that are not totally eliminated using our de-embedding method cause deviations. The measurement probes have a length of $3 \mathrm{~cm}$ and thus fields averaged over the length of the probe will be measured instead of the field value at a certain point. Using smaller measurement probes could improve the results but those probes would be less sensitive [14, Fig. 8]. A tradeoff between resolu-

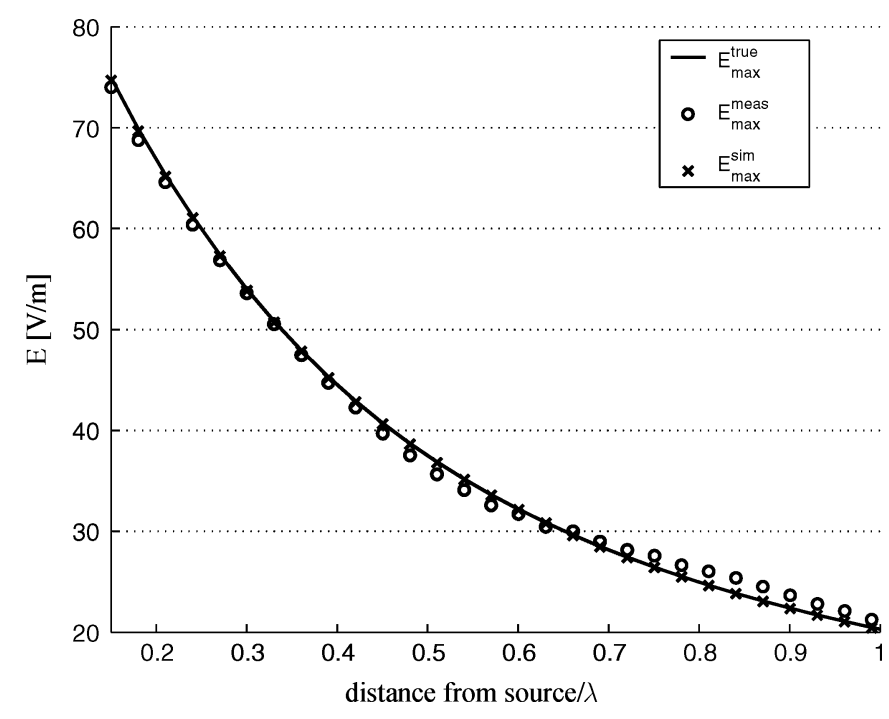

Fig. 4. Comparison of $E_{\max }$ as function of the distance from the source for free-space measurements and simulations when a $15-\mathrm{cm}$ dipole is used as Tx at $900 \mathrm{MHz}$ for $1-\mathrm{W}$ input power.

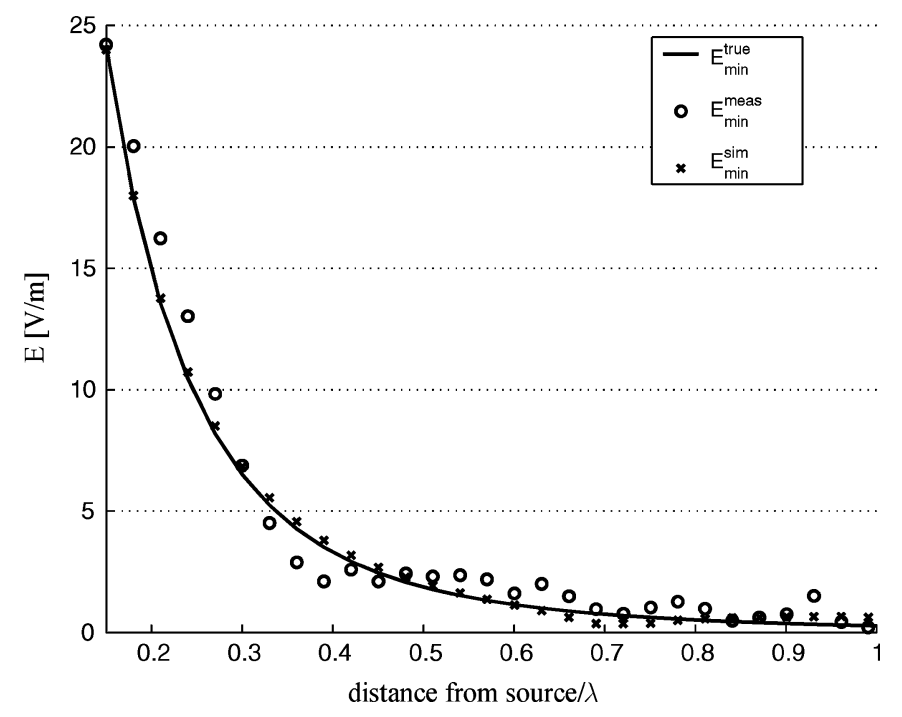

Fig. 5. Comparison of $E_{\min }$ as function of the distance from the source for free-space measurements and simulations when a $15-\mathrm{cm}$ dipole is used as Tx at $900 \mathrm{MHz}$ for $1-\mathrm{W}$ input power.

tion and sensitivity of the measurement probe has to be made. Fig. 6 shows the ellipticity $\kappa$ as function of the distance from the source/ $\lambda$ for the simulations $\left(\kappa^{\operatorname{sim}}\right)$ and measurements $\left(\kappa^{\text {meas }}\right)$ compared with the true value $\kappa^{\text {true }}$. The far-field distance of the Tx $(16 \mathrm{~cm}$ or $0.48 \lambda$ at $900 \mathrm{MHz})$ is also shown in this figure. There is again good agreement for both measurements and simulations. Up to $\lambda / 2$, the maximum and average relative deviation for the simulation of the measurements of $\kappa$ are $39.1 \%$ and $19.4 \%$, respectively. Furthermore, the average deviations $\Delta_{V}$ and $\Delta_{R}$ are, respectively, -61.3 and $-42.2 \mathrm{~dB}$ for the simulation of the measurements. The average deviations $\Delta_{V}$ and $\Delta_{R}$ are, respectively, -8.8 and $-5.6 \mathrm{~dB}$ for the NWA measurements compared with the NEC simulations. 


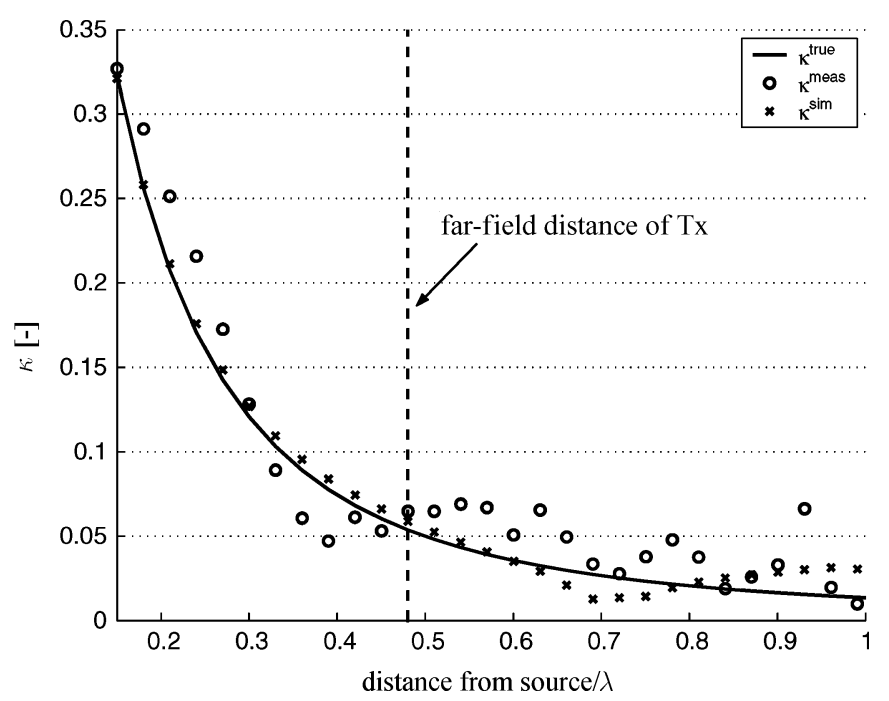

Fig. 6. Comparison of $\kappa$ as function of the distance from the source for freespace measurements and simulations when a $15-\mathrm{cm}$ dipole is used as Tx at 900 $\mathrm{MHz}$ for $1-\mathrm{W}$ input power.

Figs. 4-6 show that for both simulations and measurements very good results can be obtained compared to the results of field measurements described in the literature [3]-[5], [28]. These figures also show that the determination of the semi-major axis is more accurate than the determination of the semi-minor axis for both simulations and measurements due to the much smaller values of the semi-minor axis and the fact that $E_{\min }$ is more sensitive to measurement errors than is $E_{\max }$. To validate this, we add normally distributed noise to each of the six simulated amplitudes ( $A_{i}$ and $B_{i}, i=1,2,3$ ) of each measurement point. The magnitude of the noise is equal to $5 \%$ of the value of each of the six amplitudes of the measurement point. The average deviation of $\chi_{\max }$ then increases from $0.2 \%$ to $1.8 \%$ while the average deviation of $\chi_{\min }$ increases from $4.5 \%$ to $42.6 \% . E_{\min }$ is thus more sensitive to measurement errors. In combination with the smaller values of $E_{\min }$ this explains the larger deviations $\chi_{\min }$ in comparison to $\chi_{\max }$.

\section{LAB ApPlicAtion OF the Method With SA}

Using a lab setup, we experimentally validate the extraction of the polarization ellipse of the electric field using SA measurements with the polarization ellipse derived from the three measured complex components of $\mathrm{S}_{21}$.

\section{A. Configuration}

The configuration for SA measurements is shown in Fig. 1 and the practical implementation of the lab setup is described in Section III-A. We surround the measurement setup with absorbers to minimize the reflections. The measurements are thus not executed in an anechoic chamber. The Tx is a $15-\mathrm{cm}$ long dipole (about $\lambda / 2$ at $900 \mathrm{MHz}$ ) radiating at $900 \mathrm{MHz}$. The measurement probe is the $3-\mathrm{cm}$ long dipole. The centers of the Tx and measurement probe are positioned at the same height. The measurements are performed with a spatial grid of $1 \mathrm{~cm}$, smaller than $\lambda / 10=3.3 \mathrm{~cm}$ at $900 \mathrm{MHz}$. The measurements are per-

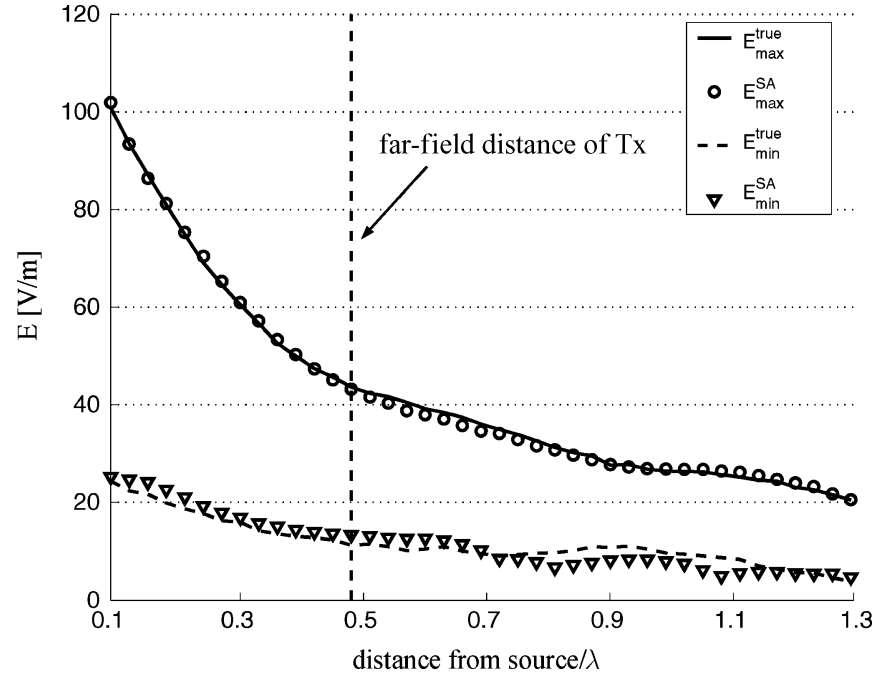

Fig. 7. Comparison of $E_{\max }$ and $E_{\min }$ as function of the distance from the source using the SA and the NWA when a $15-\mathrm{cm}$ dipole is used as Tx at 900 $\mathrm{MHz}$ for $1-\mathrm{W}$ input power.

formed from $0.1 \lambda$ to $1.3 \lambda$ from the Tx. The measurements are performed with an HP8561B spectrum analyzer (SA) with a frequency range from $50 \mathrm{~Hz}$ to $6.5 \mathrm{GHz}$. We use (4) for the determination of the amplitude components with the SA.

To obtain magnitudes and phases of the true field, we use the Rohde \& Schwarz ZVR network analyzer for the lab application. Only three components have to be measured with the NWA. The configuration for NWA measurements is shown in Fig. 3. To determine the magnitudes and phases of the three orthogonal field components with the network analyzer, the $\mathrm{S}_{21}$-parameters of two-port circuits are measured for each of the three components. With the phases of the three $\mathrm{S}_{21}$-parameters and (8) both the magnitudes and phases of the true field can be obtained. These true values will be compared in Section V-B with the values obtained using the SA and the method of Section II.

\section{B. Comparison of Results}

Fig. 7 compares the determination of $E_{\max }$ and $E_{\min }$ using the SA and NWA for 1-W input power as function of the distance from the source $/ \lambda$ at $900 \mathrm{MHz}$. Fig. 7 shows that the extracted values $E_{\max }$ and $E_{\min }$ agree well with the measurements with the NWA. Because of the reflections, $E_{\text {min }}$ does not approach zero for larger distances in contrary to the free-space situation. The far-field distance of $16 \mathrm{~cm}$ or $0.48 \lambda$ at $900 \mathrm{MHz}$ is also shown in this Fig. 7. The maximum and the average of the relative deviation $\chi_{\max }$ (true $=$ NWA measurement) are, respectively, only 0.4 and $0.2 \mathrm{~dB}(4.4$ and $1.9 \%)$. The extraction of the minimum field value $E_{\text {min }}$ delivers good results: The maximum and the average of the relative deviation $\chi_{\min }$ are, respectively, 2.2 and $1.3 \mathrm{~dB}$ (29.3 and 15.9\%). These are all small deviations compared to the measurement uncertainties published in the literature [3]-[5], [28]. The relative deviation $\chi_{\min }$ is higher than $\chi_{\max }$ due to the smaller values of $E_{\min }$. These deviations are again due to the measurement errors of the SA and NWA, calibration errors, positional and rotational errors, 


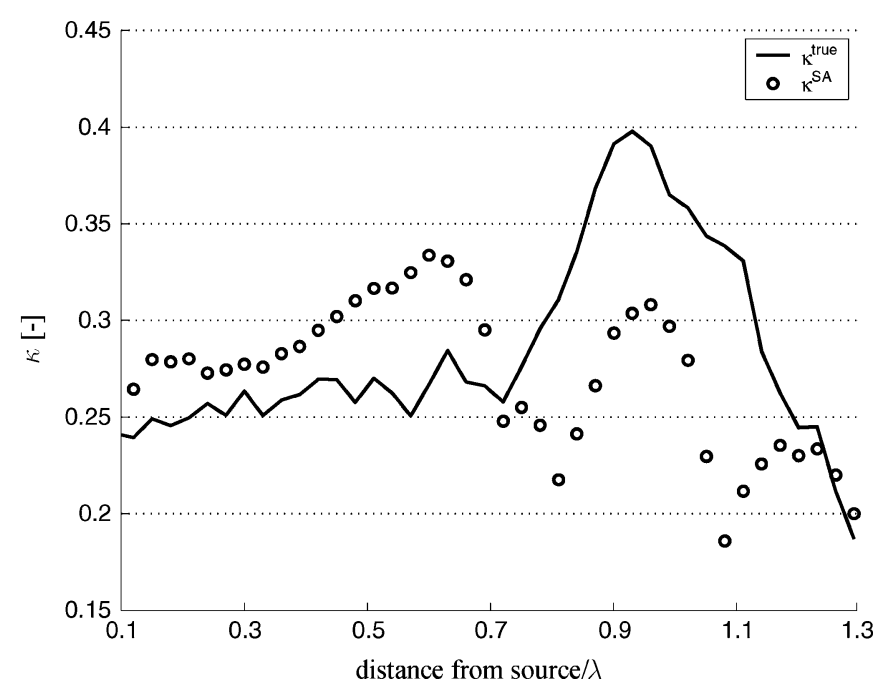

Fig. 8. Comparison of the ellipticity $\kappa$ as function of the distance from the source using the SA and using the NWA when a $15-\mathrm{cm}$ dipole is used as Tx at $900 \mathrm{MHz}$.

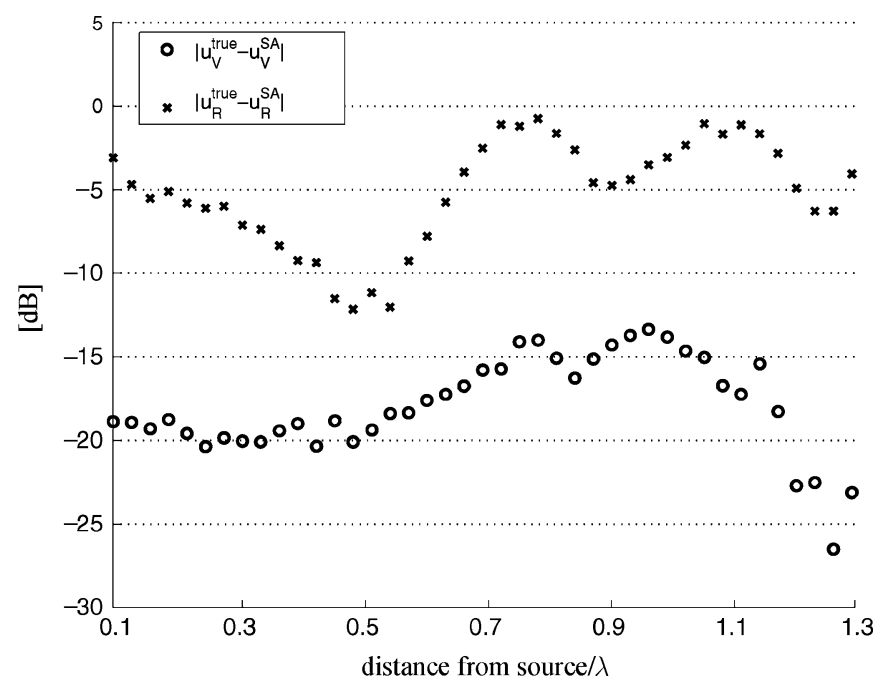

Fig. 9. Comparison of $\Delta_{V}$ and $\Delta_{R}$ using the SA and using the NWA when a $15-\mathrm{cm}$ dipole is used as Tx at $900 \mathrm{MHz}$.

and the nonperfect identical probes. Fig. 8 shows the ellipticity $\kappa$ as function of the distance from the source/ $\lambda$. The smaller $\kappa$ the more linearly the field is polarized. Fig. 9 shows $\Delta_{V}$ and $\Delta_{R}$. The average deviations of $\Delta_{V}$ and $\Delta_{R}$ are, respectively, -17.9 and $-5.1 \mathrm{~dB}$. We can conclude that the extraction of polarization ellipse delivers acceptable results. The semi-major axis can be more accurately extracted than the semi-minor axis.

Finally, we show that this method is also applicable for other frequencies. We use the $15-\mathrm{cm}$ dipole as Tx at $1800 \mathrm{MHz}$. The measurements are performed from $0.6 \lambda$ to $2.7 \lambda$ from the Tx. At $1800 \mathrm{MHz}$ the far-field distance of the Tx is 27 $\mathrm{cm}(1.62 \lambda)$. The measurement probe is again the $3-\mathrm{cm}$ dipole. The spatial grid of the measurements is $1 \mathrm{~cm}$, smaller than $\lambda / 10=1.7 \mathrm{~cm}$ at $1800 \mathrm{MHz}$. Fig. 10 shows the extraction of $E_{\max }$ and $E_{\min }$ as function of the distance from the source divided by $\lambda$ at $1800 \mathrm{MHz}$. This figure shows that again, a good agreement is obtained between the extraction with the SA and

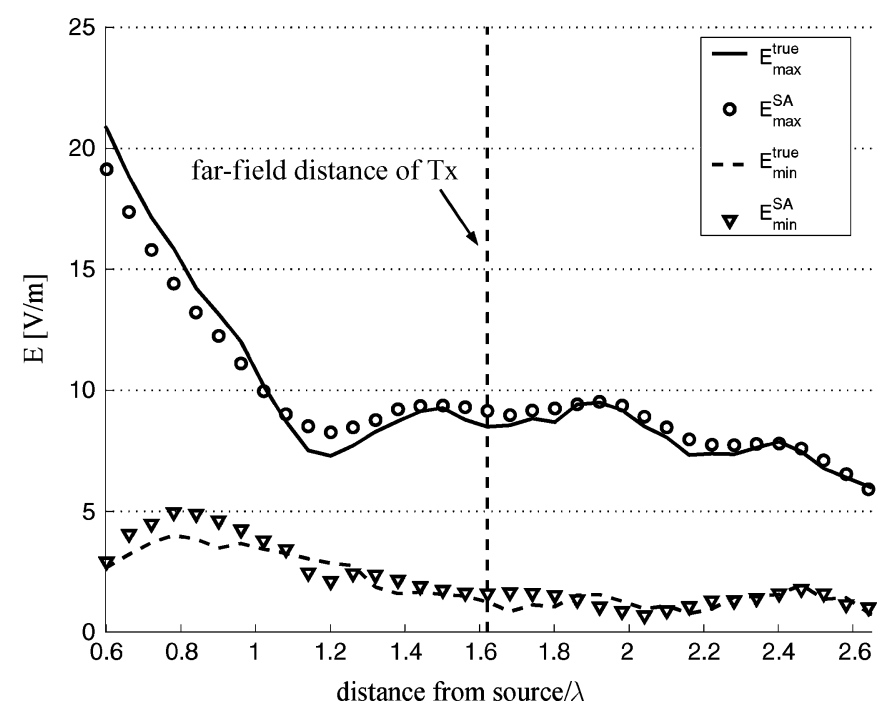

Fig. 10. Comparison of $E_{\max }$ and $E_{\min }$ as function of the distance from the source using the SA and using the NWA when the $15-\mathrm{cm}$ dipole is used as Tx at $1800 \mathrm{MHz}$ for $1-\mathrm{W}$ input power.

the NWA measurements. The average values of $\chi_{\max }$ and $\chi_{\min }$ are, respectively, 0.5 and $1.5 \mathrm{~dB}$. The average deviations $\Delta_{V}$ and $\Delta_{R}$ are, respectively, -23.2 and $-11.5 \mathrm{~dB}$.

\section{OUtdoor ApPlication OF THE METHOD With SA}

In this section, we describe an extension and adaptation of practical outdoor measurements for compliance testing of antennas [3]-[7]. We will determine six magnitudes and obtain both the magnitudes and the polarization of the field real time at a measurement site using our low-cost measurement method. Knowing the incident field allows to more accurately determine the actual SAR using a model of the human body. The measurement setup for these measurements is described in Section III-B (see Fig. 2). The purpose of the measurements is to compare the field values with proposed safety limits (e.g., ICNIRP guidelines [1]). We investigate two frequency bands: the FM band and GSM900 MHz band.

\section{A. Description of FM and GSM Measurements}

For the FM band, we use a frequency span of $21 \mathrm{MHz}$ and a center frequency of $98 \mathrm{MHz}$. For the GSM band, we use a span of $25 \mathrm{MHz}$ and a center frequency of $947.5 \mathrm{MHz}$. We assume that during the measurement time all signals in the measurement band reach a maximum. To measure the maximum level of each signal in time, the maximum-hold setting of the SA is used.

The FM measurement is performed at a rural area in the environment of Tielt, Belgium, and the measurement of the GSM signal is performed in the city of Ghent, Belgium. We perform the FM measurement at a rural area because we want to show that when the field and polarization do not vary much with the height, the actual SAR can be determined more accurately. In a rural area, when the angle of incidence $\psi$ is small (see Fig. 11, $\psi$ smaller than $10^{\circ}$ ) and when the frequency is low enough (lower than about $130 \mathrm{MHz}$ e.g., valid for FM frequencies), 


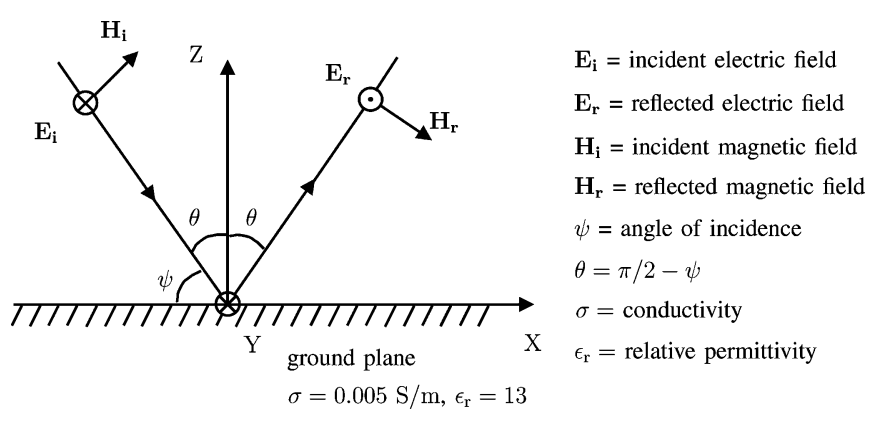

Fig. 11. Reflection of a TE-wave on a ground plane.

the variation of $X_{\max }$ and $X_{\min }(X=E$ or $H)$ as function of the height can be assumed small. This can easily be found by plane-wave analysis on a ground plane in free space (see Fig. 11 for transverse electric or TE polarization analog for transverse magnetic or TM polarization) and will be discussed in Sections VI-B and Sections VI-C. The FM antennas are mounted at a height of $290 \mathrm{~m}$ and the measurement is performed in line of sight (LOS) at $3300 \mathrm{~m}$ from the FM antenna to obtain a small angle of incidence (about $5^{\circ}$ ). The GSM antennas are mounted at a height of $64 \mathrm{~m}$ and the measurement is performed at $140 \mathrm{~m}$ from the antenna. The measurements are thus performed in the far field of the antennas. Fig. 12(a) and (b) show a typical SA measurement of the power spectrum of $A_{1}$ for the FM and GSM signal at $125 \mathrm{~cm}$ above the ground as function of the frequency using the maximum-hold mode of the SA.

We determine $X_{\text {tot }}, X_{\max }, X_{\min }(X=E$ or $H)$, and $\kappa$ as function of the height from $75 \mathrm{~cm}$ to $1.75 \mathrm{~m}$. This range corresponds with the height of the head and trunk of an average man. For the GSM signal, we look at the $\mathrm{BCCH}$ channel at 951.7 MHz. For the FM signal, we investigate the signal at 100.1 $\mathrm{MHz}$ because this signal delivers the highest total fields. At 951.7 MHz the reference level $L_{\mathrm{E}}$ of the electric field and $L_{\mathrm{H}}$ of the magnetic field for general public exposure are, respectively, $42.4 \mathrm{~V} / \mathrm{m}$ and $0.11 \mathrm{~A} / \mathrm{m} \mathrm{[1]}$. At $100.1 \mathrm{MHz}$ the reference level $L_{\mathrm{E}}$ of the electric field and $L_{\mathrm{H}}$ of the magnetic field for general public exposure are, respectively, $28 \mathrm{~V} / \mathrm{m}$ and $0.073 \mathrm{~A} / \mathrm{m}$ [1].

\section{B. Polarization Extraction for FM and GSM Antenna Fields}

The amplitudes of the electric and magnetic field components are determined using (4). Using $A_{i}$ and $B_{i}(i=1,2,3)$ we then extract the relative phases with the method described in Section II.

Fig. 13 shows the ratio of the magnitudes of the total electric and magnetic field $E_{\text {tot }} / H_{\text {tot }}$ for the FM and GSM signal. This figure shows that $E_{\text {tot }} / H_{\text {tot }}$ is not a constant equal to $377 \Omega$, because the measurements are not performed in a free-space environment due to reflections and thus constructive and destructive interference of the electric and magnetic field [29]. For $\mathrm{FM}, E_{\text {tot }} / \mathrm{H}_{\text {tot }}<377 \Omega$, thus, the magnetic field will deliver the most restrictive conditions. For the GSM signal, $E_{\text {tot }} / \mathrm{H}_{\text {tot }}$ varies much more (higher frequency). For frequencies above $300 \mathrm{MHz}$ (e.g., GSM frequencies), only the electric field is mostly considered [3]-[7], [29], [30]. We will therefore further

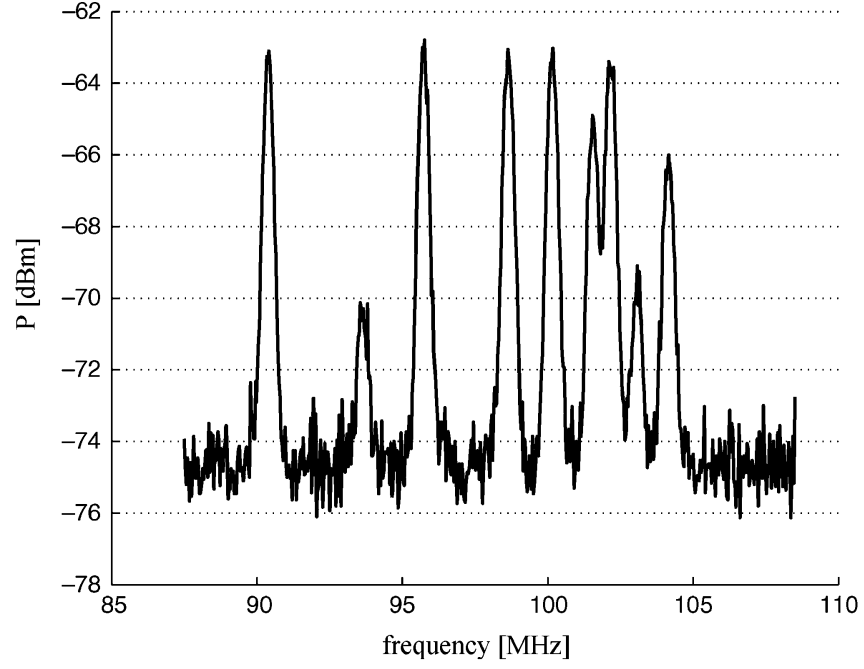

(a)

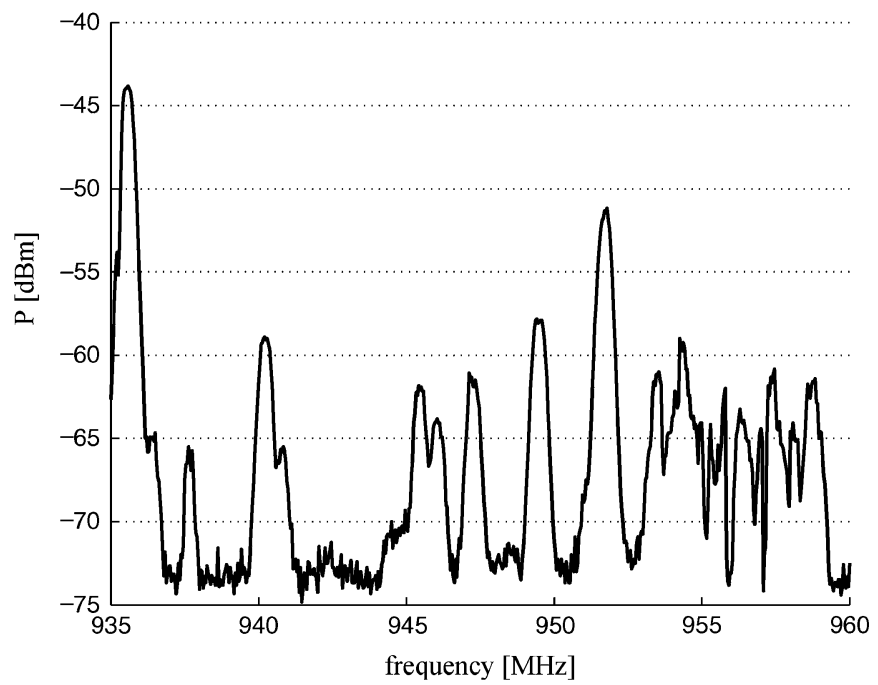

(b)

Fig. 12. Power spectrum of one component of (a) FM electromagnetic fields measured with split-shield loop antenna and (b) GSM electromagnetic fields measured with a conical dipole antenna using the $300-\mathrm{kHz}$ resolution filter of the SA.

investigate the magnetic field for the FM signal and the electric field for the GSM signal [29], [30].

The variation of $X_{\mathrm{tot}}, X_{\max }$, and $X_{\min }(X=H$ for FM and $X=E$ for GSM) as function of the height is shown in Figs. 14 and 15 , showing that the variation of the FM signal is much smaller than the variation of the GSM signal as function of the height due to the much lower frequency of the FM signal and due to fewer reflections (rural area). These figures also show that the field values are below the reference levels. Usually, $E_{\text {tot }}$ or $H_{\text {tot }}$ are compared with the reference levels [3]-[7]. Thus using this classical method [3]-[7], the maximum GSM signal at $951.7 \mathrm{MHz}(0.12 \mathrm{~V} / \mathrm{m})$ is about 350 times below the reference level of $42.4 \mathrm{~V} / \mathrm{m}$ and the maximum FM signal at $100.1 \mathrm{MHz}(0.36 \mathrm{~mA} / \mathrm{m})$ is about 200 times below the reference level of $73 \mathrm{~mA} / \mathrm{m}$.

Fig. 16 shows $\kappa_{H}$ (i.e., $\kappa$ for the magnetic field) for the FM signal at $100.1 \mathrm{MHz}$. In addition, the theoretical value of $\kappa_{H}$ 


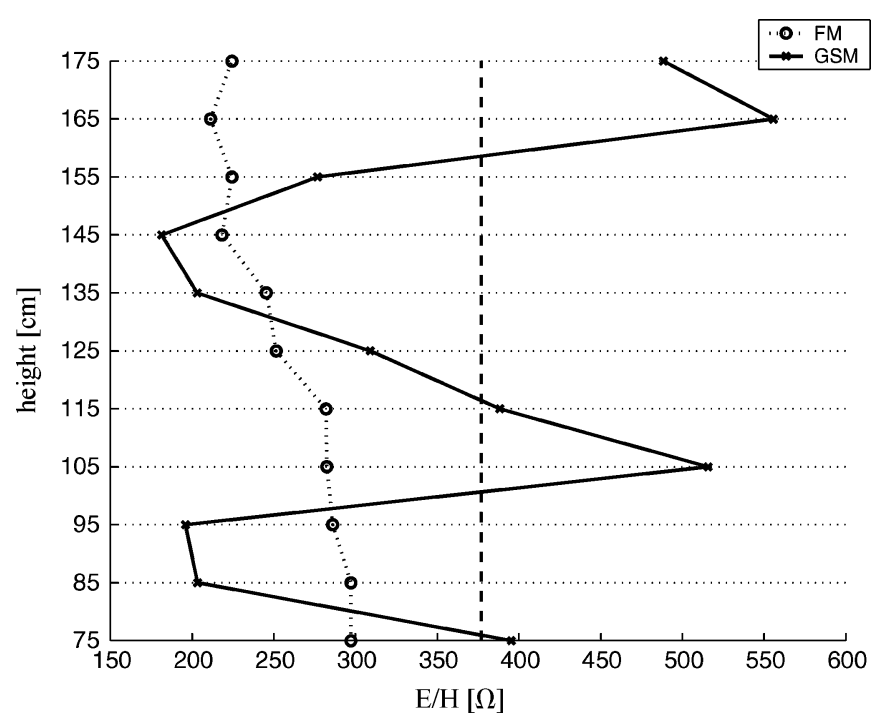

Fig. 13. E/H for the investigated FM and GSM signal.

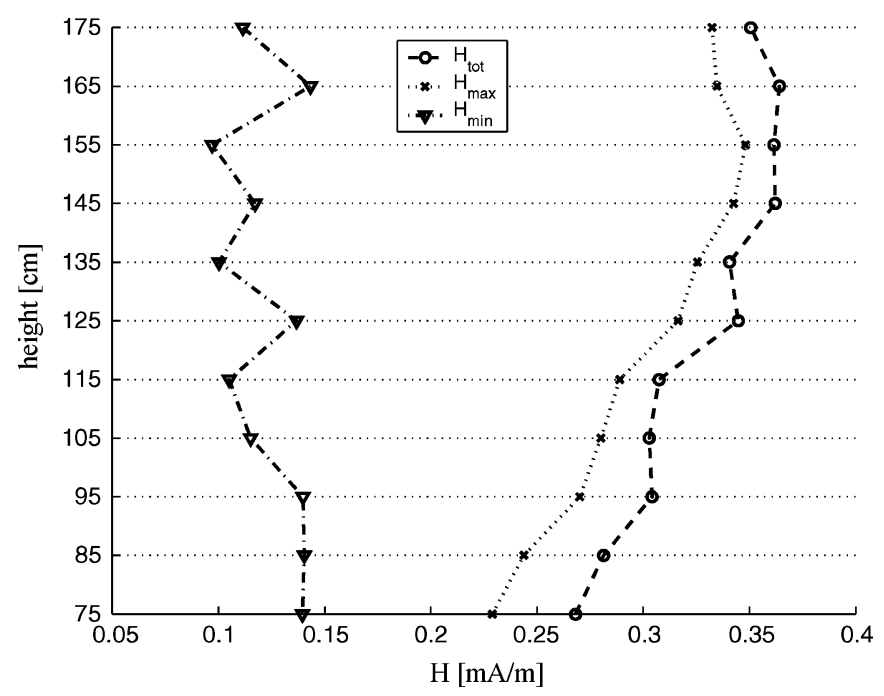

Fig. 14. $H_{\mathrm{tot}}, H_{\mathrm{max}}$, and $H_{\min }$ of the investigated FM signal as function of the height above the ground.

obtained from a plane-wave excitation model on a ground plane is shown in this figure and will be discussed in Section VI-C.

\section{Incident Field Determination for FM Base Station Antennas}

For the determination of the actual SAR using a simple model consisting of a limited number of plane waves, the GSM signal varies too much with the height. Therefore, we will now investigate the magnetic field of the FM signal as function of the height at $100.1 \mathrm{MHz}$ (see Fig. 14).

Because the measurement is performed in line of sight and in the far field of the FM antenna in a rural area, we can model the field (with magnitude and polarization of the field determined with our method, see Fig. 11) as the sum of incident plane waves on an "average" ground (dielectric parameters $\sigma=0.005$ $\mathrm{S} / \mathrm{m}$ and $\epsilon_{r}=13$ [31], [32], NEC-Win-Pro). This plane-wave excitation is then used as incident field on to the phantom model

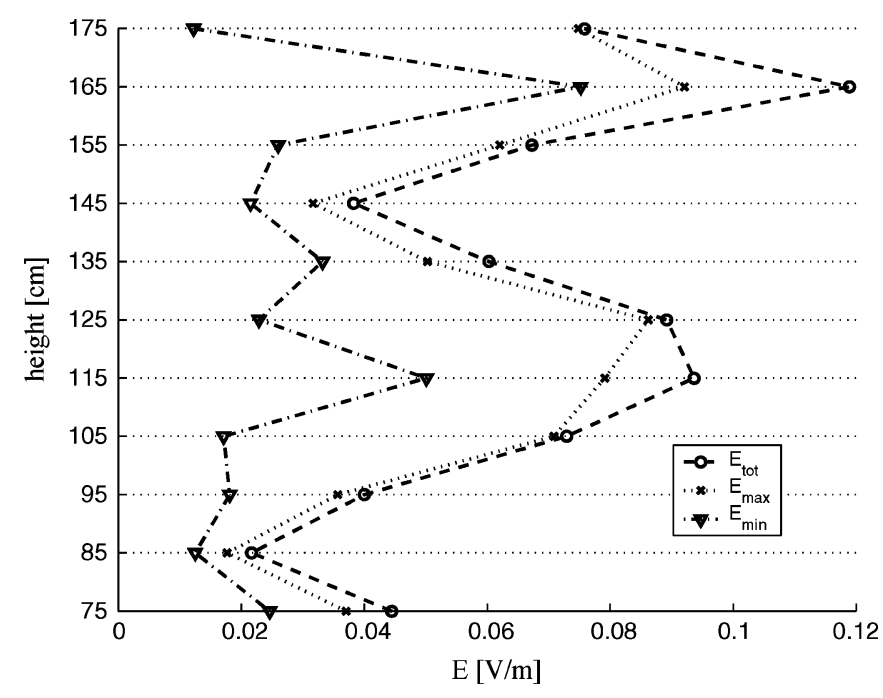

Fig. 15. $E_{\mathrm{tot}}, E_{\max }$, and $E_{\min }$ of the investigated GSM signal as function of the height above the ground.

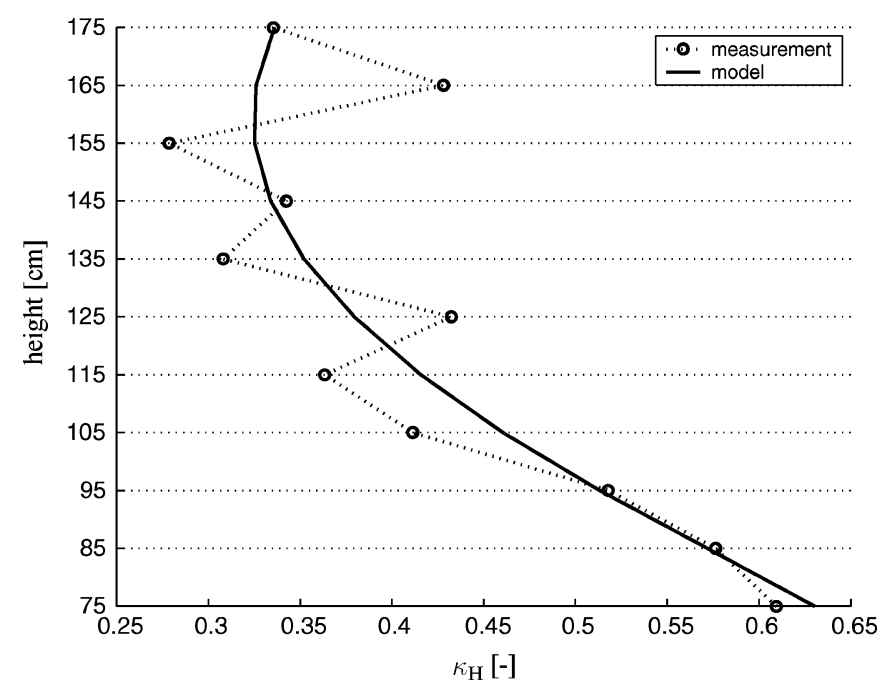

Fig. 16. Measured and theoretical value of $\kappa_{H}$ of the investigated FM signal as function of the height above the ground.

in a FDTD electromagnetic simulation. In this way we can obtain a more accurate estimate of the true SAR.

As model for the incident field we use a combination of two plane waves (TE and TM) on an "average ground." By fitting the model to the measurements using a Nelder-Mead simplex minimization method we obtain the magnitudes and angles of incidence of the plane waves. Using more plane waves in the model does not deliver a substantial benefit and augments the calculation time in the FDTD tool. Fig. 16 shows the measured value of $\kappa_{H}$ and the theoretical value obtained with the simple plane-wave excitation model. Fig. 17 compares model and measurement for $H_{\mathrm{tot}}, H_{\mathrm{max}}$, and $H_{\mathrm{min}}$. These figures show that theory and measurement correspond reasonably well. The average deviations of $\Delta_{V}$ and $\Delta_{R}$ are, respectively, -7.6 and $-8.4 \mathrm{~dB}$ (over all measurement points). Thus we obtain an acceptable correspondence between model and measurement. Deviations are caused by additional reflections of the environment, 


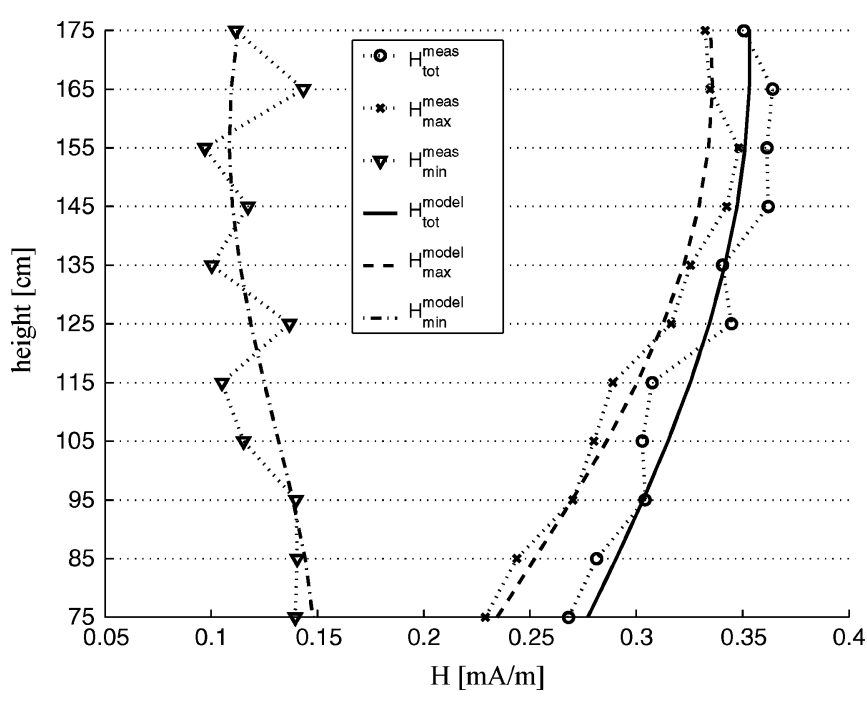

Fig. 17. Measured and theoretical value of $H_{\mathrm{tot}}, H_{\max }$, and $H_{\min }$ of the investigated FM signal as function of the height above the ground.

positional, and rotational errors and measurement errors of the SA.

\section{Whole-Body SAR for Exposure From FM Antennas}

Up to now only comparison with the reference levels could be performed using outdoor measurements. Using our measurement method, we can more accurately obtain the actual SAR and compare it with the basic restrictions. The procedure we use is the following. First, we select an appropriate phantom model. Next, we fit the plane-wave model to the data $\left(H_{\text {tot }}, H_{\max }, H_{\min }, \kappa, \ldots\right)$ obtained from our measurement of the FM signals. Using the fit we obtain the angle of incidence $\psi$ and the magnitude of the different incident plane waves (see Section VI-C and Fig. 11). Then, we use this incident plane-wave excitation model as incident field in an FDTD electromagnetic simulation tool using the phantom models standing on the average ground. Finally, we compare the whole-body SAR obtained from the FDTD simulation with the basic restrictions and define an additional safety factor $F$ (compared to the classical methods) for the FM-fields as follows:

$$
F=\frac{L_{\mathrm{SAR}} / \text { whb SAR }}{\left(L_{H} / H_{\mathrm{tot}}\right)^{2}}
$$

where

$L_{\mathrm{SAR}} \quad$ basic restriction for the whole-body SAR for the general public $(0.08 \mathrm{~W} / \mathrm{kg}$ at $100.1 \mathrm{MHz})$;

whb SAR whole-body SAR for the actual incident field,

$L_{H} \quad$ reference level of the magnetic field for the general public $(0.073 \mathrm{~A} / \mathrm{m}$ at $100.1 \mathrm{MHz})$;

$H_{\text {tot }} \quad$ magnitude of the total magnetic field.

We use in (9) the square of the ratio $L_{H} / H_{\text {tot }}$ because the SAR is proportional with the input power while the fields are proportional to the square root of the power. The factor $F$ shows for this configuration how many times the determined SAR value is more below the basic restriction than the magnetic field is below its reference level. The whole-body SAR is considered here because the whole-body SAR will deliver more restrictive conditions for this far-field (plane-wave) situation than the localized SAR [1], [33], [34].

We investigate different phantom models for this study: a realistic heterogeneous phantom model for an adult human, a homogeneous large spheroid model for this adult man, a homogeneous spheroid model for an average adult, and homogeneous spheroid models for children of five and ten years [35]. In this way, we can find out if the whole-body SAR is dependent on the age. For the realistic heterogeneous model of a man we use the model of the "Visible Human Project," which has been developed at Brooks Air Force Base Laboratories [36]. Fig. 18 shows the models standing on the ground, the dimensions and weight of the phantoms used for this study. The dimensions of the spheroids are obtained from [35].

The FDTD simulations are performed at the FM frequency 100.1 MHz. The dielectric parameters of the realistic model are those of human tissues for the investigated frequency of 100.1 MHz. For the homogeneous spheroid phantoms we use as relative permittivity $\epsilon_{r}=66$ and conductivity $\sigma=0.71 \mathrm{~S} / \mathrm{m}$ (dielectric parameters of muscle at $100.1 \mathrm{MHz}$ ). The density $\rho$ is $1000 \mathrm{~kg} / \mathrm{m}^{3}$. Since there are no sufficient data in the literature of the dielectric properties of children, we use the same dielectric parameters derived from Gabriel's data [37]-[39]. The size of the FDTD cell varies from $1 \mathrm{~mm}$ to $1 \mathrm{~cm}$.

Table I shows the whole-body $\mathrm{SAR}$, the ratio $L_{\mathrm{SAR}} /$ whb SAR, and the additional safety factor $F$ for the different phantoms for the incident plane-wave excitation obtained from our method. Table I shows that the SAR values are far below the basic restriction: the whole-body SAR is about $65 \times 10^{3}-250 \times$ $10^{3}$ lower than the basic restriction of $0.08 \mathrm{~W} / \mathrm{kg}$ for the wholebody SAR for the general public [1]. The whole-body SAR of the large adult spheroid phantom $(0.32 \mu \mathrm{W} / \mathrm{kg})$ and the average adult spheroid phantom $(0.42 \mu \mathrm{W} / \mathrm{kg})$ are slightly lower than the whole-body SAR of the heterogeneous realistic model $(0.43 \mu \mathrm{W} / \mathrm{kg})$. Use of a homogeneous phantom model may result in lower SAR values than a heterogeneous and anatomically realistic model like we mentioned in [33], but at $100.1 \mathrm{MHz}$, this difference is limited for the whole-body SAR in case of spheroid phantoms. The values of the whole-body SAR are larger for the child phantoms than for the adult phantoms because of their smaller dimensions (resonance frequency closer to $100.1 \mathrm{MHz}$ than the one of adult phantoms [35]) but the SAR values are still far below the basic restrictions. Using (9), we obtain the additional safety factor $F$ in Table I. For the adult phantoms, $F$ varies from 4.6 to 6.3 . For the 10 -year old phantom $F=1.8$ and for the 5 -year old child phantom $F=1.6$. This shows that the SAR values for the investigated configuration and phantoms are about 1.6-6.3 times more below the basic restrictions than the field values are below the reference levels for this configuration. Thus, using our method based on amplitude measurements only we are able to determine more accurately the actual SAR using different models of a human and the polarization of the incident field. For outdoor measurements, we can now compare the SAR values with the basic restrictions instead of only comparing the field values with their reference levels like the classical methods do [3]-[7]. 


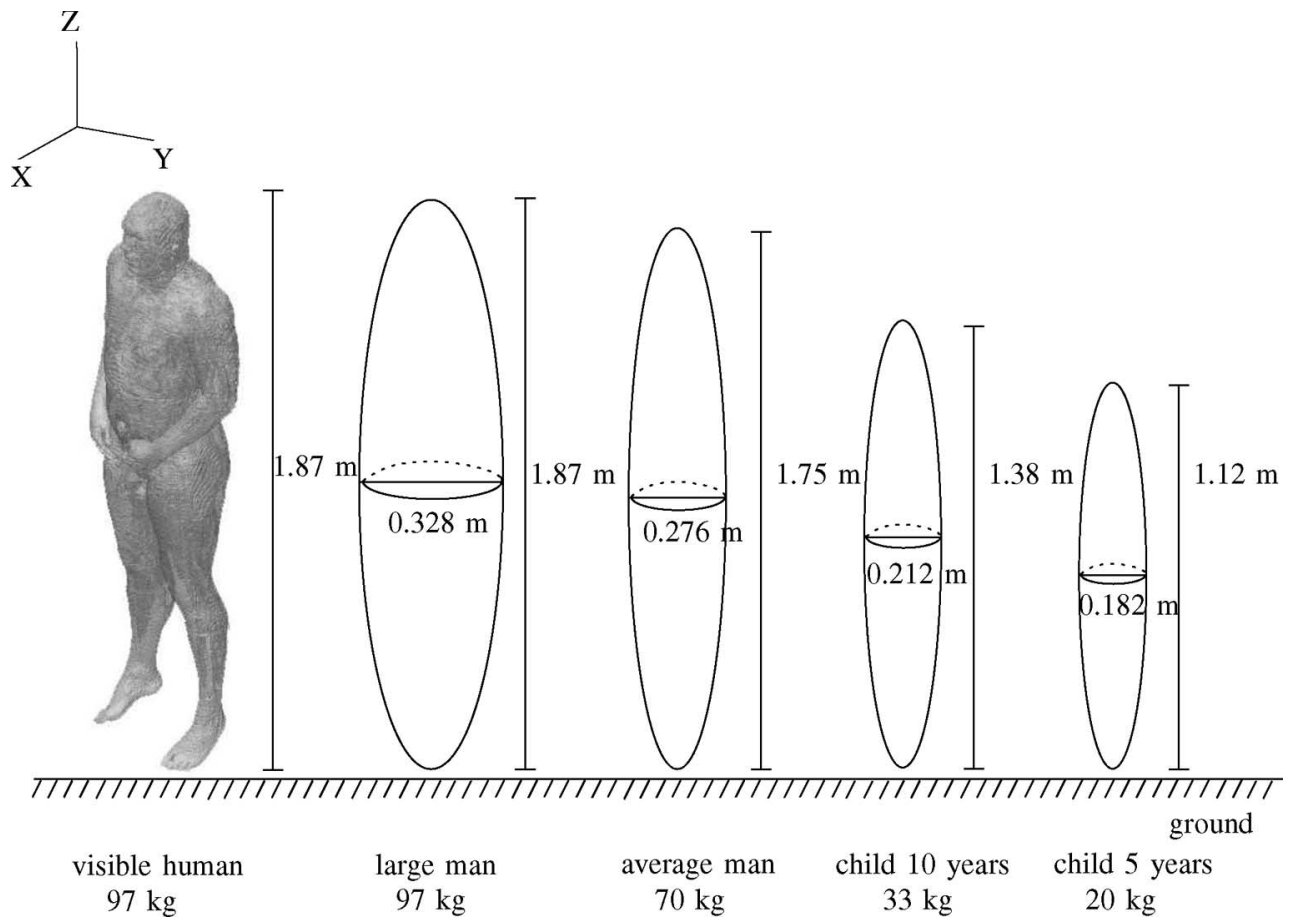

Fig. 18. Different human model phantoms for calculation of the whole-body SAR.

TABLE I

SAR RESULTS FOR AN FM SIGNAL AT 100.1 MHz OBTAINEd USING OUR MEASUREMENT METHOD AND THE FIELD MODEL INCIDENT ON DIFFERENT PHANTOMS

\begin{tabular}{|c||c|c|c|c|c|}
\hline Phantom & Human & Large man & Average man & Child 10 year & Child 5 year \\
\hline \hline Type & Visible human & Spheroid & Spheroid & Spheroid & Spheroid \\
\hline Length $[\mathrm{m}]$ & 1.87 & 1.87 & 1.75 & 1.38 & 1.12 \\
\hline Weight $[\mathrm{kg}]$ & 97 & 97 & 70 & 33 & 20 \\
\hline Whb SAR $[\mu \mathrm{W} / \mathrm{kg}]$ & 0.43 & 0.32 & 0.42 & 1.09 & 1.23 \\
\hline$L_{\mathrm{SAR}} /$ whb SAR & $184 \mathrm{E} 3$ & $252 \mathrm{E} 3$ & $189 \mathrm{E} 3$ & $73 \mathrm{E} 3$ & $65 \mathrm{E} 3$ \\
\hline$F$ & 4.57 & 6.26 & 4.71 & 1.83 & 1.62 \\
\hline
\end{tabular}

\section{CONCLUSION}

In this paper, we presented a low-cost measurement method for the extraction of the polarization of electromagnetic fields of antennas for outdoor measurements using six magnitude measurements only. We are able to determine immediately the magnitudes and polarization of the electromagnetic field at a measurement site and obtain a substantial reduction in computing time over methods that use nonlinear optimization. To validate the measurement method, we compared the extraction using the SA with NWA measurements. We obtained a good agreement: for the electric field quantities $E_{\max }$ and $E_{\min }$ an average deviation of, respectively, $0.2 \mathrm{~dB}$ and $1.3 \mathrm{~dB}$ at $900 \mathrm{MHz}$ is reported. The deviations of $E_{\min }$ are larger than those of $E_{\max }$. We have applied the method for FM and GSM signals and have shown that the method can be practically used for outdoor measurements. By retrieving the polarization of the incident field we are able to determine more accurately the actual SAR for outdoor measurements and compare the SAR with the basic restrictions instead of only comparing the field values with the reference levels. We have therefore defined an additional safety factor for the SAR in a realistic model of a man and in spheroid models of adults and children.

Furthermore, the applicability of the method to UMTS like signals is discussed in this paper. Applying the method of this paper with other phantoms and different postures (standing and sitting) will result in different absorption values and is a subject for future research.

\section{REFERENCES}

[1] International Commission on Non-ionizing Radiation Protection (ICNIRP), "Guidelines for limiting exposure to time-varying electric, magnetic, and electromagnetic fields (up to $300 \mathrm{GHz}$ ") Health Phys., vol. 74, pp. 494-522, 1998.

[2] H. M. C. H. Durney, M. F. Iskander, and C. C. Johnson, "An empirical formula for broad-band SAR calculations of prolate spheroidal models of humans and animals," IEEE Trans. Microw. Theory Tech., vol. MTT-27, no. 8, pp. 758-763, Aug. 1979.

[3] C. Olivier and L. Martens, "A practical method for compliance testing of base stations for mobile communications with exposure limits," in Proc. IEEE Antennas Propag. Soc. Int. Symp., Boston, MA, Jul. 2001, pp. 64-67.

[4] _ , "Measurements of exposure to electromagnetic radiation around GSM base stations in belgium," in Proc. 23rd Ann. Meeting Bioelectromagn. Soc., St. Paul, MN, Jun. 2001, pp. 102-104.

[5] G. Neubauer, W. Giczi, and G. Schmid, "An optimized method to determine exposure due to GSM base stations applied in the city of Salzburg," in Proc.24th Ann. Meeting Bioelectromagn. Soc., Quebec, QC, Jun. 2002, pp. 46-47.

[6] G. Neubauer, H. Haider, M. Riederer, and R. Coray, "Measurement methods and legal requirements for exposure assessment next to GSM base stations," in Proc. EMC Zurich Symp., Zurich, Switzerland, Feb. 2003, pp. 143-148.

[7] H. Lehmann, P. Fritschi, and B. Eicher, "Indoor measurements of the electrical field close to mobile phone base stations," presented at the 27th Triennial General Assembly of the International Union of Radio Science, Maastricht, The Netherlands, Aug. 2002, Paper 2112. 
[8] D. B. Nguyen, "An algebraic method to reconstruct the relative phases and polarization of a complex vector in $\mathrm{n}$ dimensions based on $3(\mathrm{~N}-1)$ amplitude measurements," IEEE Trans. Instrum. Meas., vol. 40, no. 6, pp. 966-971, Dec. 1991.

[9] D. B. Nguyen and S. Berntsen, "The reconstruction of the relative phases and polarization of the electromagnetic field based on amplitude measurements," IEEE Trans. Microw. Theory Tech., vol. 40, no. 9, pp. 1805-1811, Sep. 1992.

[10] D. B. Nguyen and J. W. Strohbehn, "The polarization of a complex vector in higher dimensions," IEEE Trans. Antennas Propag., vol. 39, no. 4, pp. 555-556, Apr. 1991.

[11] O. P. Ghandi and M. S. Lam, "An on-site dosimetry system for safety assessment of wireless base stations using spatial harmonic components," IEEE Trans. Antennas Propag., vol. 51, no. 4, pp. 840-847, Apr. 2003.

[12] G. Lazzi and O. P. Gandhi, "A mixed fdtd-integral equation approach for on-site safety assessment in complex electromagnetic environments," IEEE Trans. Antennas Propag., vol. 51, no. 4, pp. 1830-1836, Dec. 2003.

[13] W. Joseph, L. Verloock, and L. Martens, "An accurate low-cost measurement technique for occupational exposure assessment of base station antennas," Electron. Lett., vol. 39, no. 12, pp. 886-887, Jun. 2003.

[14] - "The influence of the measurement probe on the evaluation of electromagnetic fields," IEEE Trans. Electromagn. Compat., vol. 43, no. 2, pp. 339-349, May 2003.

[15] J. A. A. Smith, F. German, and B. Pate, "Calculation of site attenuation from antenna factors," IEEE Trans. Electromagn. Compat., vol. EMC-24, no. 3, pp. 301-316, Aug. 1982.

[16] J. A. A. Smith, "Standard-site method for determining antenna factors," IEEE Trans. Electromagn. Compat., vol. EMC-24, no. 3, pp. 316-322, Aug. 1982.

[17] W. Joseph and L. Martens, "An improved method to determine the antenna factor," IEEE Trans. Instrum. Meas., vol. 54, no. 1, pp. 252-257, Feb 2005.

[18] L. M. C. Carobbi and L. Chiosi, "The high-frequency behavior of the shield in the magnetic-field probes," in Proc. IEEE EMC, vol. 1, Washington, DC, Aug. 2000, pp. 35-40.

[19] C. Carobbi and L. Millanta, "Analysis of the common-mode rejection in the measurement and generation of magnetic fields using loop probes," IEEE Trans. Instrum. Meas., vol. 53, no. 2, pp. 514-523, Apr. 2004.

[20] ANSI C63.5, "American National Standard for Electromagnetic Compatibility - Radiated Emission Measurements in Electromagnetic Interference (EMI) Control-Calibration of Antennas (9 kHz to $40 \mathrm{GHz})$,", 1998.

[21] W. Joseph and L. Martens, "A robust, fast and accurate deconvolution algorithm for EM-field measurements around gsm and umts base stations with a spectrum analyser," IEEE Trans. Instrum. Meas., vol. 51, no. 6, pp. 1163-1169, Dec. 2002.

[22] C. Olivier and L. Martens, "Optimal settings for narrow-band signal measurements used for exposure assessment around GSM base stations," IEEE Trans. Instrum. Meas., vol. 54, no. 1, pp. 311-317, Feb. 2005.

[23] — "Measurement issues for electromagnetic exposure assessment around UMTS base stations," in Proc. Bioelectromagnetics 2005, to be published.

[24] - "Theoretical derivation of the stochastic behavior of a WCDMA signal measured with a spectrum analyzer GSM base stations," IEEE Trans. Instrum. Meas., to be published.

[25] _ _ "Optimal settings for frequency-selective measurements used for the exposure assessment around UMTS base stations," IEEE Trans. Instrum. Meas., to be published.

[26] N. N. M. Mbonjo, J. Streckert, A. Bitz, V. Hansen, A. Glasmachers, S. Gencol, and D. Rozic, "Generic UMTS test signal for RF bioelectromagnetic studies," Bioelectromagnetics, vol. 25, no. 6, pp. 415-4252, Sep. 2004.

[27] R. Yaguie, A. Ibars, and L. Martinez, "Analysis and reduction of the distortions induced by time-domain filtering techniques in network analyzers," IEEE Trans. Instrum. Meas., vol. 47, no. 4, pp. 930-934, Aug. 1998.

[28] E. Nicolas, D. Lautru, M. F. Wong, and J. Wiart, "Specific absorption rate assessments based on a selective isotropic measuring system for electromagnetic fields," IEEE Trans. Electromagn. Compat., vol. 50, no. 2, pp. 397-401, Apr. 2001.

[29] C. Olivier and L. Martens, "Electromagnetic field measurements in multipath exposure conditions," presented at the Int. NIR workshop \& Symposium, Seville, Spain, May 2004.

[30] IEEE Standard for Safety Levels with Respect to Human Exposure to Radio Frequency Electromagnetic Fields, $3 \mathrm{kHz}$ to $300 \mathrm{GHz}$, IEEE Std. C95.1, 1999.
[31] A. Priou and J. A. Kong, Dielectric Properties of Heterogeneous Materials, PIER 6 Progress in Electromagnetic Research. New York: Elsevier, 1992.

[32] A. Sihvola, Electromagnetic Mixing Formulas and Applications. London, U.K.: IEE, 1999.

[33] W. Joseph and L. Martens, "Safety factor for the determination of occupational exposure in phantom model," Electron. Lett., vol. 39, no. 23, pp. 1663-1664, Nov. 2003.

[34] _ "Determination of the correction factor for occupational exposure compliance evaluation using different homogeneous phantoms," in Proc. 26th Ann. Meeting Bioelectromagn. Soc., Washington, DC, Jun. 2004, pp. 92-95.

[35] H. M. C. H. Durney and M. F. Iksander, Radiofrequency Radiation Dosimetry Handbook, 4th ed. San Antonio, TX: USAF School of Aerospace Medicine, Brooks Air Force Base, 1997.

[36] J. Ziriax, D. L. Blanc, P. Mason, and W. D. Hurt, "Finite-difference timedomain for personal computers," in Proc. 21st Ann. Meeting Bioelectromagn. Soc., Long Beach, CA, Jun. 1999, p. 57.

[37] C. Gabriel, "Compilation of the dielectric properties of body tissues at RF and microwave frequencies," Brooks Air Force Base, San Antonio, TX, Tech. Rep. AL/OE-TR-1996-0037, 1996.

[38] J. Wang and O. Fujiwara, "Comparison and evaluation of electromagnetic absorption characteristics in realistic human head models of adult and children for $900 \mathrm{MHz}$ mobile telephones," IEEE Trans. Microw. Theory Tech., vol. 51, no. 3, pp. 966-971, Mar. 2003.

[39] L. Martens and C. Olivier, "COST281 project on mobile communications and children," presented at the the Int. Electromagnetic Field Project, Sensitivity of Children to Electromagnetic Fields, Istanbul, Turkey, Jun. 2004

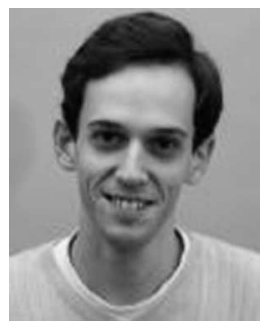

Wout Joseph was born in Ostend, Belgium, on October 21, 1977. He received the M.Sc. degree in electrical engineering and the Ph.D. degree in accurate assessment of electromagnetic fields and absorption in the neighbourhood of GSM and broadcast antennas from the Ghent University, Gent, Belgium, in July 2000 and March 2005, respectively.

From September 2000 to March 2005, he was a Research Assistant at the Department of Information Technology (INTEC), Ghent University. Since April 2005, he has been a Postdoctoral Researcher for the Interdisciplinary Institute for BroadBand Technology (IBBT-Ugent/INTEC) Ghent University, and his interests are electromagnetic field measurements, propagation for wireless communication systems, antennas, and calibration.

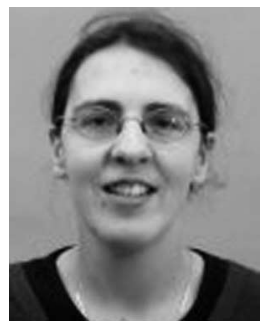

Leen Verloock was born in Eeklo, Belgium, on November 15, 1979. She received the M.S. degree in electronics engineering from the Katholieke Hogeschool Ghent, Gent, Belgium, in 2001.

In 2001, she joined the Department of Information Technology (INTEC), Ghent University, Gent, Belgium, where she is currently working as a Technical and Research Assistant in the Wireless and Cable Research Group. She is working on propagation effects for wireless systems and the influence of the user on this propagation. She assists Ph.D. students with their research about modeling and measuring the propagating fields of wireless systems.

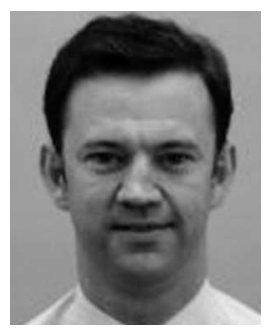

Luc Martens (M'92) was born in Gent, Belgium, on May 14, 1963. He received the M.Sc. degree in electrical engineering and the Ph.D. degree in development of a multi-channel hyperthermia system: electromagnetic modelling of applicators, generator design, and estimation algorithms for thermometry from Ghent University, Gent, in July 1986 and December 1990, respectively.

From September 1986 to December 1990, he was a Research Assistant at the Department of Information Technology (INTEC), Ghent University. Since January 1991, he has been a Member of the permanent staff of the Interuniversity MicroElectronics Center (IMEC), Ghent, and is responsible for the research on experimental characterization of the physical layer of telecommunication systems at INTEC. Since April 1993, he has been a Professor of electrical applications of electromagnetism at Ghent University. 